\title{
Bolometric luminosity variations in the luminous blue variable AFGL2298*
}

\author{
J. S. Clark ${ }^{1}$, P. A. Crowther ${ }^{2}$, V. M. Larionov ${ }^{3,4}$, I. A. Steele ${ }^{5}$, B. W. Ritchie ${ }^{1,6}$, and A. A. Arkharov ${ }^{7}$ \\ 1 Department of Physics and Astronomy, The Open University, Walton Hall, Milton Keynes, MK7 6AA, UK \\ e-mail: jsc@star.ucl.ac.uk \\ 2 Department of Physics \& Astronomy, University of Sheffield, Sheffield, S3 7RH, UK \\ Astronomical Institute of St. Petersburg University, Petrodvorets, Universitetsky pr. 28, 198504 St. Petersburg, Russia \\ Isaac Newton Institute of Chile, St. Petersburg Branch, Russia \\ Astrophysics Research Institute, Liverpool JMU, Twelve Quays House, Egerton Wharf, Birkenhead, CH41, 1LD, UK \\ 6 IBM United Kingdom Laboratories, Hursley Park, Winchester, Hampshire, S021 2JN, UK \\ Pulkovo Astronomical Observatory, 196140 St. Petersburg, Russia
}

Received 21 April 2009 / Accepted 27 August 2009

ABSTRACT

\begin{abstract}
Aims. We characterise the variability in the physical properties of the luminous blue variable AFGL 2298 (IRAS 18576+0341) between 1989-2008.

Methods. In conjunction with published data from 1989-2001, we have undertaken a long term (2001-2008) near-IR spectroscopic and photometric observational campaign for this star and utilise a non-LTE model atmosphere code to interpret these data.

Results. We find AFGL 2298 to have been highly variable during the two decades covered by the observational datasets. Photometric variations of $\geq 1.6$ mag have been observed in the JHK wavebands; however, these are not accompanied by correlated changes in near-IR colour. Non-LTE model atmosphere analysis of 4 epochs of $K$ band spectroscopy obtained between 2001-7 suggests that the photometric changes of AFGL 2298 were driven by expansion and contraction of the stellar photosphere accompanied by comparatively small changes in the stellar temperature $\left(\Delta T_{*} \sim 4.5 \mathrm{kK}\right)$. Unclumped mass loss rates throughout this period were modest and directly comparable to those of other highly luminous (candidate) LBVs. However, the main finding of this analysis was that the bolometric luminosity of AFGL 2298 appears to have varied by at least a factor of 2 between 1989-2008, with it being one of the most luminous stars in the Galaxy during maximum. Comparison to other LBVs that have undergone non bolometric luminosity conserving "eruptions" shows such events to be heterogeneous, with AFGL 2298 the least extreme example. These results - and the diverse nature of both the quiescent LBVs and associated ejecta - may offer support to the suggestion that more than one physical mechanism is responsible for such behaviour.
\end{abstract}

Key words. stars: evolution - stars: early-type - stars: supergiants - stars: individual: AFGL 2298

\section{Introduction}

Luminous blue variables (LBVs) represent a transitional state in the evolution of massive stars between main sequence and hydrogen depleted Wolf-Rayets and are characterised by significant photometric and spectroscopic variability (e.g. Lamers 1987; Humphreys \& Davidson 1994). Two characteristic modes have historically been identified: (i) 1-2 mag excursions on year timescales at constant bolometric luminosity $\left(L_{\mathrm{bol}}\right)$ and (ii) giant ( $\geq 2 \mathrm{mag}$ ) eruptions, during which the luminosity of the star increases, but for which the timescales are currently uncertain due to their rarity.

Recently, LBVs have been the subject of renewed interest. With the likely downwards revision of Main Sequence mass loss rates it has been suggested that $\eta$ Car-like giant eruptions play a key role in stripping the $\mathrm{H}$ rich mantle from post-MS stars prior to the WR phase (Smith \& Owocki 2006). Moreover, some anomalously faint type IIn SNe have been hypothesised to be LBVs in an eruptive phase rather than core-collapse events (e.g. Humphreys et al. 1999; Goodrich et al. 1989). Finally, several lines of evidence have been advanced to suggest that LBVs may be the immediate progenitors of a subset of type II SN,

^ Based on observations made at the European Southern Observatory, Paranal, Chile under programs 077.C-0207 and 079.D-0769. including some of the most luminous type IIn events ever observed (e.g. Kotak \& Vink 2006; Smith et al. 2007; Gal-Yam et al. 2007; Trundle et al. 2007; Pastorello et al. 2007), in turn leading to the suggestion that the occurrence of $\eta$ Car-like eruptive events may warn of an imminent SN (e.g. Smith et al. 2007; Woosley et al. 2007).

Given these possibilities, an understanding of the physics leading to both modes of LBV variability is urgently required. Unfortunately, such a goal is hampered by the evident rarity of LBVs and their attendant outbursts. Only two examples of non $L_{\text {bol }}$ conserving eruptions have been spectroscopically studied HD 5980 (Koenigsberger 2004) and NGC 2363-V1 (Drissen et al. 2001) - both of which occurred in low metallicity environments. However, with the expansion of IR astronomy over the past decade, a number of new candidate LBVs have been identified within the Galaxy via both imaging and spectroscopy (e.g. Clark et al. 2005b, and references therein). Of these, the presence of high mass circumstellar nebulae surrounding a subset of B hypergiants is particularly suggestive of enhanced mass loss in the past, possibly associated with giant eruptions (Ueta et al. 2001; Clark et al. 2003b; Smith \& Owocki 2006).

AFGL 2298 (=IRAS 18576+0341), observed as part of a long term spectroscopic and photometric monitoring campaign of (candidate) LBVs, has been found to be highly vari- 


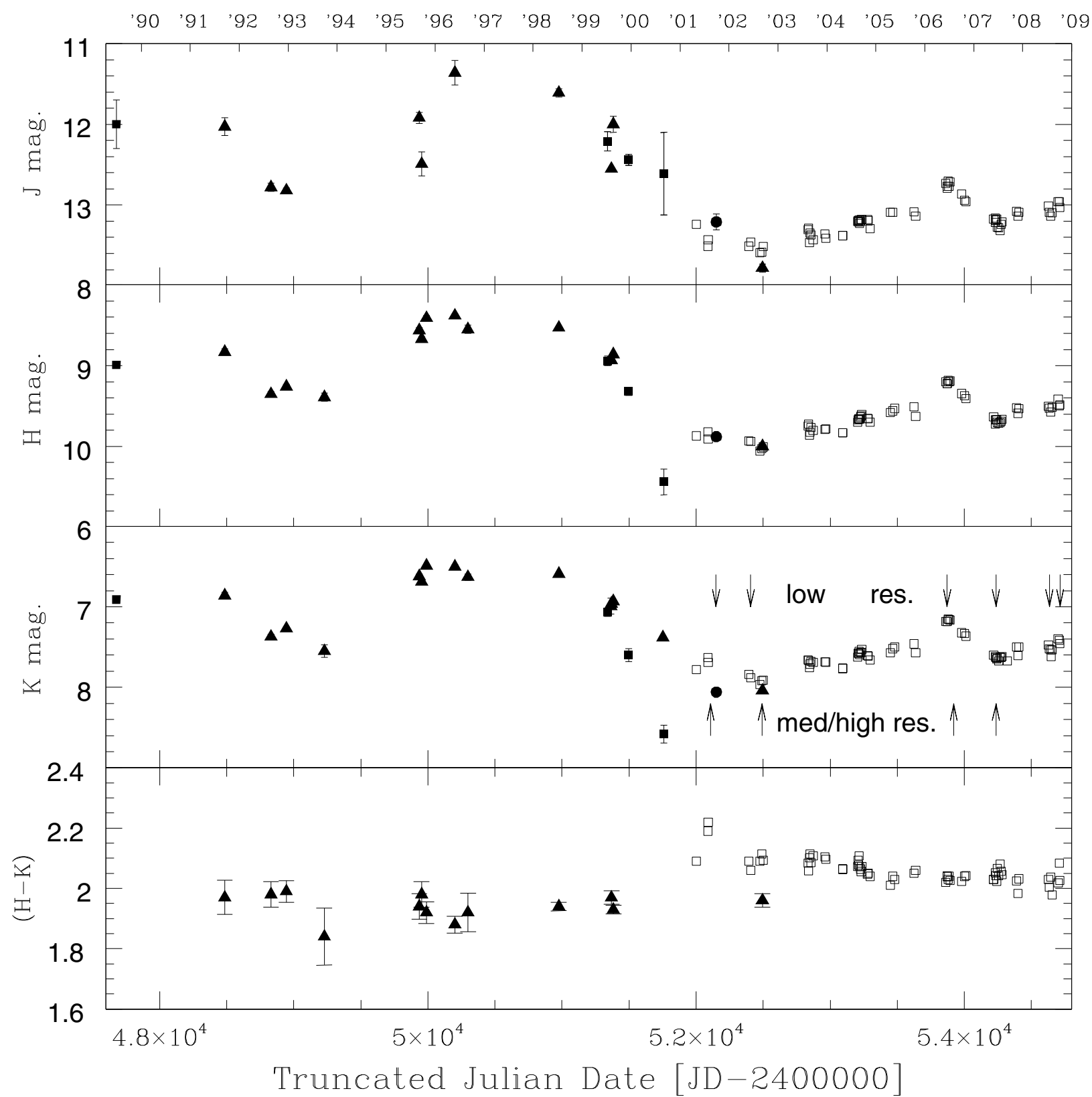

Fig. 1. JHK band lightcurves and $(H-K)$ colour index for AFGL 2298 spanning $\sim$ two decades. Data are from Jimenez-Esteban et al. (2006; filled triangles), Ueta et al. (2001; filled squares), Pasquali \& Comeron (2002; open circles) and Clark et al. (2005b) and this work (open squares). Where not shown, error-bars are smaller than the size of the respective symbols. The arrows in the $K$ band panel indicate the times at which the spectroscopic observations were made, with the low, medium and high resolution observations made with the AZT-24 1.1 m, UKIRT/CGS4 and the VLT/ISAAC respectively (see Sect. 2).

able (Clark et al. 2003a). Spectroscopic analysis of near-IR observations suggested significant changes in stellar radius and temperature between 2001-2002, indicative of an LBV excursion. In this paper we update the results of this effort, encompassing an extended lightcurve and additional medium resolution spectroscopy and an accompanying analysis with the nonLTE model atmosphere code CMFGEN (Hillier \& Miller 1998, 1999). Finally, in light of these results and other recent results, we discuss the observational properties of LBV variability and the implications for the formation of ejection nebulae.

\section{Data reduction}

Near-IR JHK broadband photometric observations of AFGL 2298 were obtained at the AZT-24 $1.1 \mathrm{~m}$ telescope in Campo Imperatore (Italy) from 2001 March-2008 October. The SWIRCAM $256 \times 256 \mathrm{HgCdTe}$ detector was employed, yielding a scale of 1.04 arcsec/pix, resulting in a $\sim 4^{\prime} \times 4^{\prime}$ field of view. Standard techniques of sky subtraction and flat-fielding were applied. Between two and four standards were employed for the calibration of the photometry. All standards were located within the target frames, had near-IR magnitudes between 9.9 and $11.5 \mathrm{mag}$. and were found to be constant to within \pm 0.02 mag over the course of the observations. The resultant lightcurve is presented in Fig. 1.

Spectra of AFGL 2298 have been obtained from a number of different telescopes and are summarised in Table 1, and presented in Figs. 2, 3. A description of the UKIRT/CGS4 observations and reductions may be found in Clark et al. (2003a). Long term, low resolution $(R \sim 270)$ spectroscopic monitoring was 
Table 1. Log of the spectroscopic observations between 2001-8.

\begin{tabular}{cccr}
\hline \hline Date & Telescope & $\begin{array}{c}\lambda \\
(\mu \mathrm{m})\end{array}$ & Resolution \\
\hline $27 / 06 / 01$ & UKIRT/CGS4 & $2.0-2.5$ & 800 \\
$22 / 07 / 02$ & AZT-24 1.1 m & $1.45-2.38$ & 270 \\
$06 / 08 / 02$ & UKIRT/CGS4 & $2.0-2.5$ & 800 \\
$17 / 05 / 06$ & AZT-24 1.1 m & $1.45-2.38$ & 270 \\
$30 / 06 / 06$ & VLT/ISAAC & $2.04-2.22$ & 8900 \\
$15 / 05 / 07$ & AZT-24 1.1 m & $1.45-2.38$ & 270 \\
$09 / 05 / 07$ & VLT/ISAAC & $2.04-2.22$ & 8900 \\
$16 / 06 / 08$ & AZT-24 1.1 m & $1.45-2.38$ & 270 \\
$04 / 09 / 08$ & AZT-24 1.1 m & $1.45-2.38$ & 270 \\
\hline
\end{tabular}

undertaken with the AZT-24 $1.1 \mathrm{~m}$ telescope and the IR imaging camera SWIRCAM+HK band grism - providing spectral coverage between $1.45-2.38 \mu \mathrm{m}$. Higher resolution $(R \sim 8900)$ follow up observations were subsequently made with the VLT in 2006 and 2007.

The VLT observations were made with ISAAC in the shortwavelength (SW) medium resolution (MR) mode with a narrow $0.3^{\prime \prime}$ slit. To achieve spectral coverage from $2.04-2.22 \mu \mathrm{m}$ two exposures were obtained, centred at $2.10 \mu \mathrm{m}$ and $2.16 \mu \mathrm{m}$. All data were taken with a count rate of below 10000 ADU, therefore no correction for non-linearity was necessary. Data reduction was accomplished using the FIGARO and KAPPA software packages. Note that the telluric correction was poor for the 2007 spectrum due to rapidly varying sky conditions.

\section{Results}

\subsection{Photometry}

With the addition of the data presented here and in Jimenez Esteban et al. (2006) the lightcurve for AFGL 2298 extends for $\sim 2$ decades, revealing significant near-IR variability throughout this time (Fig. 1). Our well sampled lightcurve from 2001-8 reveals two minima. The first, deeper minimum occurred in the second half of 2002 and the second in the first half of 2007, with the corresponding maximum in mid 2006. Low amplitude $(\sim 0.2 \mathrm{mag})$ variability over timescales of $\sim$ days is superimposed on the long term trends in all 3 wavebands.

In addition to these minima, the comparatively poorly sampled data set of Jimenez Esteban et al. (2006) provides evidence for a further photometric minimum between 1993-5 and a maximum between 1996-1999. The 3 minima and 2 maxima appear to have differing intensities, with a maximum peak to trough variation of $\geq 1.6$ mag observed between the 1996-1999 maximum and 2002 minimum.

In the bottom panel of Fig. 1 we plot the $(H-K)$ colour index, finding no systematic variation with either the long term secular changes or short timescale low amplitude variability during these observations. As such this behaviour differs from that demonstrated by the candidate LBVs G24.73+0.69 and G26.47+0.02 (Clark et al. 2005b; Clark et al., in prep.), where both stars became significantly redder as they brightened. Indeed the lack of colour variability associated with either rising or falling branches of the lightcurve of AFGL 2298 argues against the changes being due to episodes of enhanced mass loss ${ }^{1}$.

\footnotetext{
${ }^{1}$ Where a brightening accompanied by an increase in reddening may reflect increased emission from circumstellar material (and vice versa e.g. WR137 and WR19; Williams et al. 2001; and Veen et al. 1998), while a reduction (increase) in brightness accompanied by an increase
}

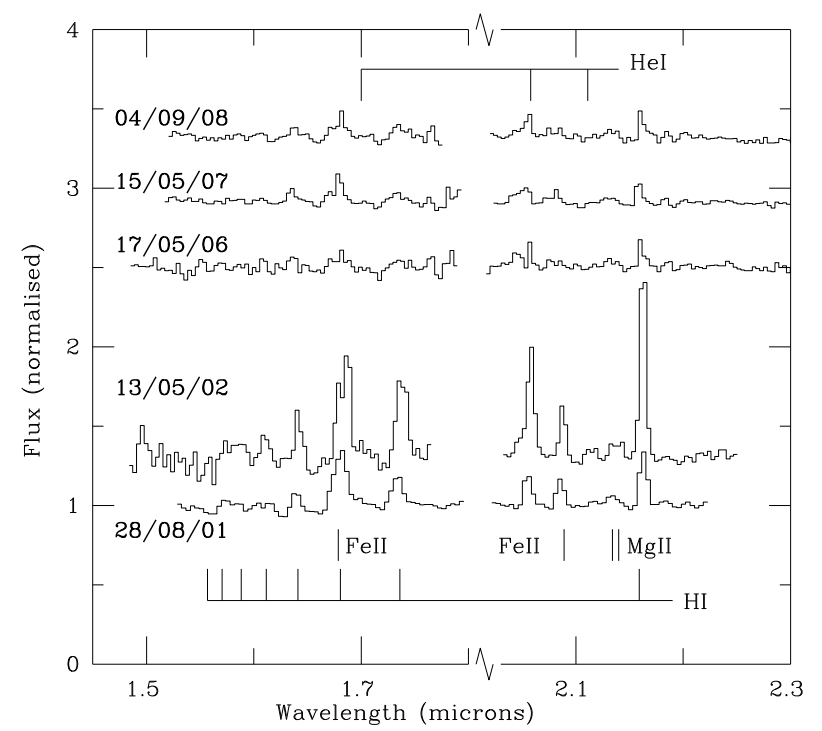

Fig. 2. Selected low resolution AZT-24 spectra of AFGL 2298 from 2002-2008. Note the significant reduction in the strengths of all emission lines between 2006-9 in comparison to 2001-2 (also see Fig. 3).

Instead, we attribute it to changes in the properties of the underlying star, although the identical near-IR colours of early $\mathrm{OB}$ stars preclude a determination of stellar temperature from these data alone.

For our spectroscopic analysis, we adopt $E(B-V)=9 \mathrm{mag}$ from Ueta et al. (2001), equating to an infrared extinction of $A_{J}=8.25, A_{H}=4.65$ and $A_{K}=2.7$, plus a distance of $10 \mathrm{kpc}$ (distance modulus $15.0 \mathrm{mag}$ ), as in Clark et al. (2003a). With this choice of infrared extinction, absolute magnitudes in the $J$, $H$ and $K$ bands differ by at most $0.1 \mathrm{mag}$, in agreement with predictions from atmospheric models (Sect. 4). No evidence was found for hot dust in any of the 4 epochs of spectroscopic observations - JHK magnitudes were well reproduced by the reddened spectral energy distributions in all cases.

\subsection{Spectroscopy}

Figure 2 presents our AZT-24 low resolution $(R \sim 270) H$ - and $K$-band spectroscopy of AFGL 22298, while low-medium resolution ( $R \sim 800-8900)$ UKIRT and VLT $K$-band spectroscopy is presented in Fig. 3. These illustrate the significant change in spectral morphology between 2001-2002 reported by Clark et al. (2003a), as well as a subsequent dramatic reduction in strength of the emission lines of all species by 2006 May, a state which has persisted for the past $\sim 2.5 \mathrm{yr}$. Unfortunately, a lack of observations between 2002 August and 2006 May prevent us localising the transition - if indeed it were a sudden event - over the course of the secular brightening of AFGL 2298 during this period (recall Fig. 1). However, following the 2006 maximum, no pronounced changes are observed in the low resolution spectra despite the subsequent photometric variability.

This is emphasised by the medium resolution VLT/ISAAC spectrum of 2006 June 30 (Fig. 3) - fortuitously obtained close to infrared maximum (Fig. 1) - which reveals a narrow emission line spectrum $\left(F W H M_{\mathrm{Br} \gamma}=70 \mathrm{~km} \mathrm{~s}^{-1}\right)$ dominated by $\mathrm{Br} \gamma$ and low excitation metallic species. Comparison to the 2002

(decrease) in reddening may be attributed to increased (decreased) extinction due to very dense circumstellar material (e.g. IRAS 160293041; Jimenez-Esteban et al. 2006). 

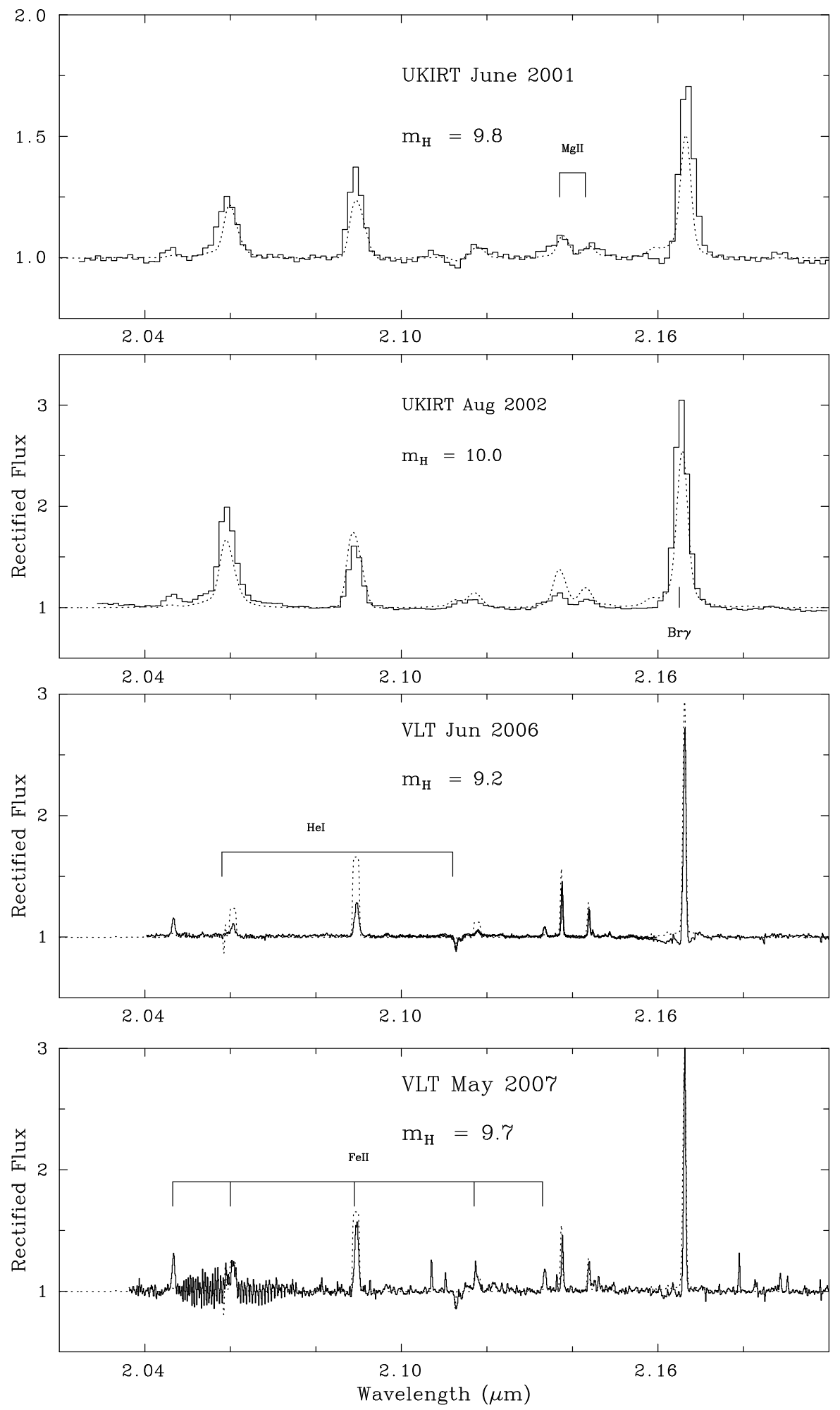

Fig. 3. Montage of 4 epochs of medium (UKIRT/CGS4: $R \sim 800$ ) and high (VLT/ ISAAC; $R \sim 8900$ ) resolution $K$ band spectroscopy of AFGL 2298 from 2001 and 2007 (solid lines) with the major transitions indicated (note that telluric subtraction was poor for the 2007 observation). The resultant spectra from the non-LTE model atmosphere analysis in Sect. 4.2 are overplotted (dotted lines). Note the different scales on the $y$-axis.
UKIRT/CGS4 spectrum obtained during the photometric minimum confirms the pronounced weakening in line emission for all species; He I emission - prominent in the 2001-2002 spectra - is entirely absent, with the He I $2.112 \mu \mathrm{m}$ transition seen in absorption.

A second VLT/ISAAC medium resolution spectrum obtained on 2007 May 9 - this time during the subsequent local photometric minimum - shows a moderate recovery in the strength of Fe II emission lines, but no significant changes in the strength of $\mathrm{Br} \gamma$ or the $\mathrm{Mg}$ II and He I lines. These changes - in particular the absence of He I emission - suggest a modest reduction in temperature after 2002 (Sect. 4.2; Table 2), although all the spectra are entirely consistent with the classification of AFGL 2298 as a cool highly luminous B supergiant throughout the 2001-9 period.

Finally, these observations reveal spatial extended emission in the Br $\gamma$ line at distances of up to 4 arcsec from the star, allowing us to infer an expansion velocity of $\pm \sim 70 \mathrm{~km} \mathrm{~s}^{-1}$ for the detached ejection nebula associated with AFGL 2298 (Fig. 4). Adopting the radial extent and mass given by Ueta et al. (2001) 
Table 2. Summary of physical and wind properties of AFGL 2298 for 2001-2007.

\begin{tabular}{lcccc}
\hline \hline Epoch & 2001 Jun. & 2002 Aug. & 2006 Jun. & 2007 May \\
\hline$J$ & 13.4 & 13.7 & 12.7 & 13.2 \\
$H$ & 9.8 & 10.0 & 9.2 & 9.6 \\
$K$ & 7.9 & 7.9 & 7.2 & 7.6 \\
$M_{H}$ & -9.85 & -9.65 & -10.45 & -10.05 \\
\hline$T_{*}(\mathrm{kK})$ & 12.5 & 15.5 & 11.0 & 11.5 \\
$R_{*}\left(R_{\odot}\right)$ & 262 & 158 & 385 & 312 \\
$T_{\text {eff }}(\mathrm{kK})$ & 11.7 & 10.9 & 10.3 & 10.9 \\
$R_{2 / 3}\left(R_{\odot}\right)$ & 300 & 320 & 444 & 353 \\
$\mathrm{BC}_{H}$ & -0.85 & -0.90 & -0.55 & -0.65 \\
$L_{*} / L_{\odot}$ & $1.5 \times 10^{6}$ & $1.3 \times 10^{6}$ & $2.0 \times 10^{6}$ & $1.5 \times 10^{6}$ \\
$\dot{M}\left(M_{\odot} \mathrm{yr}^{-1}\right)$ & $4.5 \times 10^{-5}$ & $1.2 \times 10^{-4}$ & $5.2 \times 10^{-5}$ & $4 \times 10^{-5}$ \\
$v_{\infty} \mathrm{km} \mathrm{s}^{-1}$ & 200 & 200 & 125 & 125 \\
$\mathrm{H} / \mathrm{He}$ & 5 & 5 & 5 & 5 \\
\hline
\end{tabular}

Note 1. For all epochs we have assumed $\mathrm{DM}=15, E(B-V)=9$, $A_{J}=8.25, A_{H}=4.64, A_{K}=2.69$ (from Ueta et al. 2001) and $\mathrm{H} / \mathrm{He}=$ 5.0 , while $v_{\infty} \sim 200 \mathrm{~km} \mathrm{~s}^{-1}$ was assumed for 2001-2002; these latter two parameters are given in italics. Mass-loss rates presented here are not corrected for wind clumping. We find errors of $\pm 2 \mathrm{kK}( \pm 0.2 \mathrm{mag}$ in BC), $\pm 1.5 \mathrm{kK}( \pm 0.2 \mathrm{mag})$ and $\pm 0.5 \mathrm{kK}( \pm 0.1 \mathrm{mag})$ for 2001,2002 and 2006-2007 respectively.

implies a kinematic age of $8300 \mathrm{yr}$ and consequently a time averaged mass loss rate of $1.2 \times 10^{-3} M_{\odot} \mathrm{yr}^{-1}$ during nebular formation.

\section{Physical properties of AFGL 2298}

\section{1. $1989-2001$}

In the absence of spectroscopic data, the interpretation of the photometric lightcurve of AFGL 2298 prior to 2001 is subject to uncertainties due to the fact that near-IR colours of hot OB stars are $\sim$ constant. Nevertheless, given the similar stellar luminosities, magnitude of the excursions $(\Delta J \sim 2.5$; Fig. 1 and Groh, priv. comm.) and timescale of variability ( $\sim 2-4$ yrs; Fig. 1, Spoon et al. 1994), it would appear that AG Car should serve as a suitable template for comparison to AFGL 2298. Groh (2007) studied AG Car through two full photometric cycles from 1986-2005 and found a transition from $T_{\text {eff }} \sim 24$ to $\sim 8 \mathrm{kK}$, anticorrelated with both $V$ and $J$ band magnitudes, as expected for LBV excursions.

Somewhat unexpectedly, in light of the prevailing orthodoxy, Groh et al. (2009) found that the $L_{\text {bol }}$ of AG Car was not conserved over the period of these cycles, with a reduction of $\sim 50 \%$ during photometric maximum/temperature minimum when compared to photometric minimum/temperature maximum. Similar behaviour has been observed for $\mathrm{S}$ Dor (e.g. van Genderen 2001), with Lamers (1995) interpreting the resultant deficit in radiative energy during photometric maximum as being due to the increased mechanical energy required to move and subsequently support the outer layers of the star against gravity at a greatly expanded radius.

However, AFGL 2298 was significantly cooler $\left(T_{\mathrm{eff}} \sim\right.$ $10.8 \mathrm{kK}$ ) in the 2002-2003 photometric minimum than AG Car in a comparable phase $(\sim 24 \mathrm{kK})$. Such a low temperature implies a $(H$-band) bolometric correction of $<1$ mag (Sect. 4.2$)$. If AFGL 2298 were to have mirrored the behaviour of AG Car between 1989-2001 - i.e. with periods of increasing IR brightness corresponding to a decrease in temperature (and vice versa) the magnitude of near IR variability observed $(>1.6 \mathrm{mag}$ ) would preclude such changes occuring at reduced or even constant $L_{\text {bol }}$

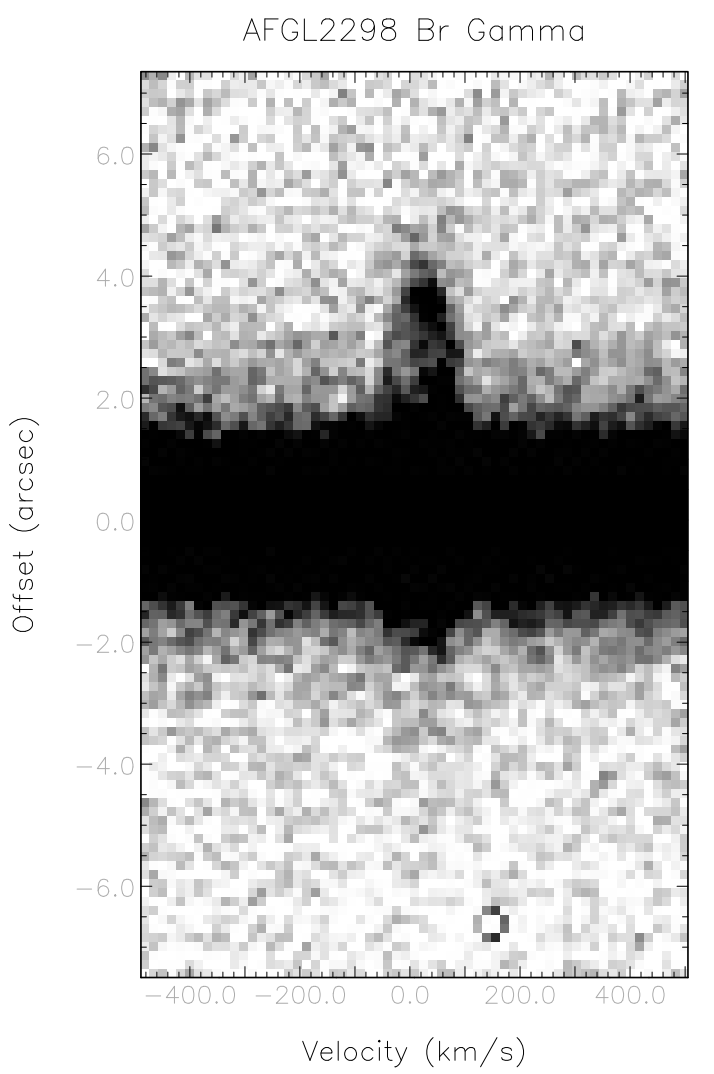

Fig. 4. Long slit spectrum of AFGL2298 centred on the Br $\gamma$ line with a North(uppermost)-South orientation. Extended, nebular emission is present up to 4 arcsec northwards of the star $(0.2 \mathrm{pc}$ for a distance of $10 \mathrm{kpc}$ ), with the elliptical morphology consistent with material expanding at up to $\pm \sim 70 \mathrm{~km} \mathrm{~s}^{-1}$. Note that the physical extent and the pronounced asymmetrical nature of the emission is consistent with the morphology of the ionised component of the nebula found from the radio continuum observations of Umana et al. (2005).

and instead would indicate that the increase in IR brightness that accompanied a reduction in stellar temperature would at least in part be due to a genuine increase in luminosity. Trivially, such a conclusion would also be drawn if the variability occurred at constant or increasing temperature. We will return to this below, upon consideration of the results of non-LTE modeling of the post 2001 data.

\section{2. $2001-2007$}

While we may not unambiguously interpret the 1989-2001 behaviour of AFGL 2298 from photometric data alone, the contemporaneous spectroscopy in the period 2001-2007 allows us to accomplish this via a non-LTE spectroscopic analysis using CMFGEN (Hillier \& Miller 1998, 1999). We explicitly follow an identical methodology to that employed in Clark et al. (2003a), in order to permit a direct comparison of all four epochs of spectroscopic observations.

\subsubsection{Atmospheric code}

CMFGEN solves the radiative transfer equation in the comoving frame, under the additional constraints of statistical and radiative equilibrium. It does not solve the momentum equation, so a density or velocity structure is required. For the supersonic part, the velocity is parameterized with a classic $\beta$-type law. 
CMFGEN incorporates line blanketing through a super-level approximation, in which atomic levels of similar energies are grouped into a single super-level which is used to compute the atmospheric structure. Our atomic model is similar to that employed by Drissen et al. (2001), including ions from $\mathrm{HI}$, He I-II, N I-II, O I-II, Mg I-II, Al II-III, Si II-III, S II-III, Ti II-III and Fe II-III. By number, the main contributors to line blanketing are Fe II-III. $\mathrm{H} / \mathrm{He} \sim 5$ by number was adopted for all models, since solely $K$-band datasets do not readily allow the determination of $\mathrm{H} / \mathrm{He}$ contents without a temperature diagnostic independent of helium. Solar abundances are taken for metals, with the exception of 5 times solar for nitrogen, as expected for chemically evolved LBVs.

Stellar temperatures, $T_{*}$, correspond to a Rosseland optical depth of 20, which are typically (up to) a few hundred degrees higher than effective temperatures, $T_{2 / 3}$, relating to a Rosseland optical depth of $2 / 3$.

We have assumed a depth-independent Doppler profile for all lines when solving for the atmospheric structure in the comoving frame, while in the final calculation of the emergent spectrum in the observer's frame, we have adopted a uniform turbulence of $20 \mathrm{~km} \mathrm{~s}^{-1}$. Incoherent electron scattering and Stark broadening for hydrogen and helium lines are adopted.

One improvement afforded by the ISAAC datasets was the means of observationally constraining the terminal wind velocity in 2006-7, which had to be estimated for the 2001-2002 data. As in Clark et al. (2003a), a $\beta=2$ velocity law was adopted for all subsequent epochs. The form of the velocity law is not expected to differ from epoch to epoch, especially between the crucial 2006 and 2007 datasets. With regard to wind clumping, this was not included for consistency with Clark et al. (2003a), although volume filling factors of $f \sim 0.1$ typically reduce massloss rates by a factor of $\sqrt{1 / f} \sim 3$.

\subsubsection{Analysis}

We derive the stellar temperature of AFGL 2298 primarily using the $K$-band He I line diagnostics. Specifically, the higher resolution 2006-7 VLT/ISAAC datasets allowed the He I $2.058 \mu \mathrm{m}$ emission line and $2.112 \mu \mathrm{m}$ absorption line to be resolved, thereby allowing significantly more robust physical parameters (e.g. He I $2.058 \mu \mathrm{m}$ and Fe II $2.060 \mu \mathrm{m}$ were blended in 2001-2002 UKIRT/CGS4 datasets).

For Jun. 2006, the simultaneous presence of strong $2.112 \mu \mathrm{m}$ absorption plus negligible $2.058 \mu \mathrm{m}$ emission occurs for a narrow range in temperatures, namely $11 \pm 0.5 \mathrm{kK}$ (equivalent to \pm 0.1 mag in Bolometric Correction; BC), as shown in the upper panel of Fig. 5. Similar conclusions were drawn for two LBVs in the Quintuplet cluster by Najarro et al. (2009). The mass-loss rate is fixed from $\mathrm{Br} \gamma$ with other $K$-band features either reproduced satisfactorily ( $\mathrm{Mg}$ II doublet), or predicted somewhat too strong (Fe II lines).

For May 2007, weak $2.112 \mu \mathrm{m}$ absorption plus weak $2.058 \mu \mathrm{m}$ P Cygni emission implies a slightly higher stellar temperature of $11.5 \pm 0.5 \mathrm{kK}$ (again reliable to $\pm 0.1 \mathrm{mag}$ in BC) - see lower panel of Fig. 5 - plus a reduced mass-loss rate from $\mathrm{Br} \gamma$. For this epoch, both $\mathrm{Mg}$ II and Fe II lines are now satisfactorily reproduced, including the Fe II feature partially blended with $2.058 \mu \mathrm{m}$.

For reference, we have also investigated the potential effect of a varying $\beta$ velocity law, for the 2007 epoch. Use of a $\beta=1$ law would favour a $0.5 \mathrm{kK}$ higher temperature, yet maintain the $H$-band bolometric correction to within 0.03 mag.
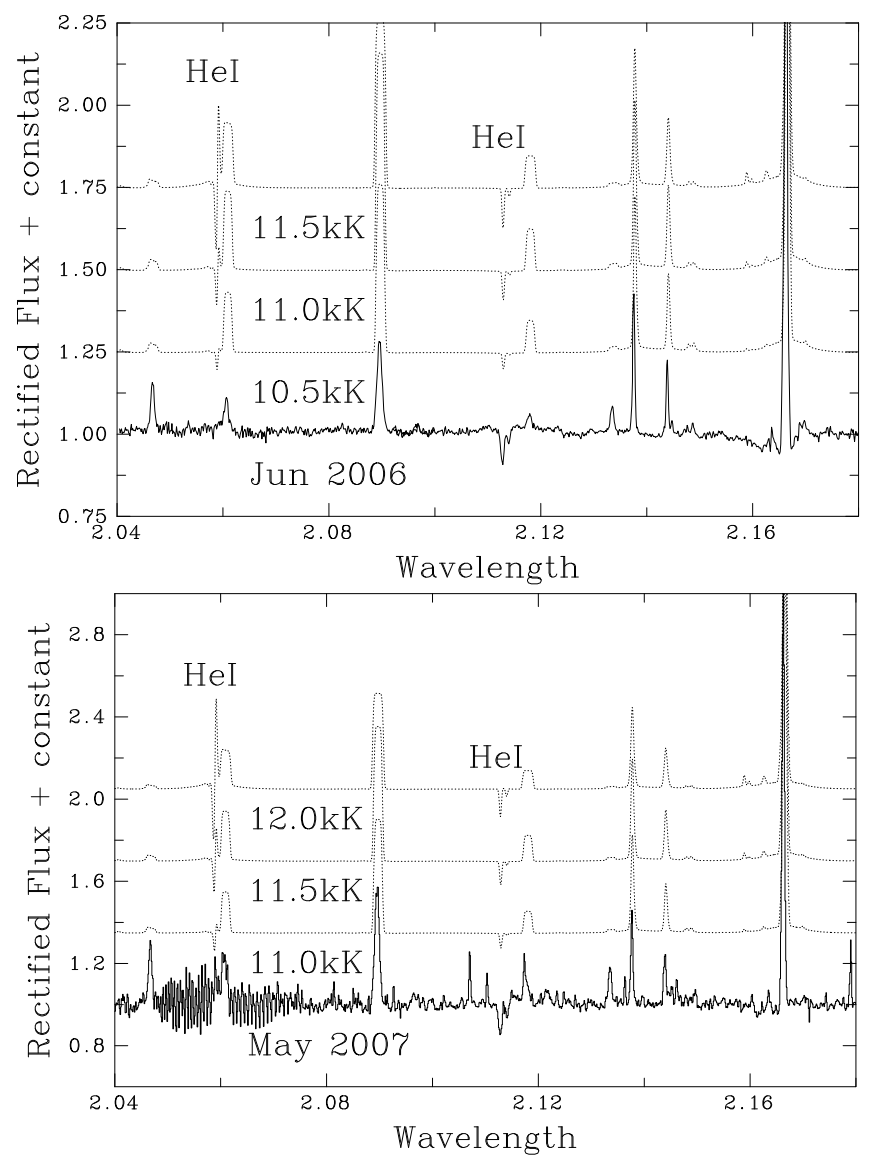

Fig. 5. VLT/ISAAC spectroscopy of AFGL 2298 for (upper panel) Jun. 2006 together with synthetic spectra for $11 \pm 0.5 \mathrm{kK}$; (lower panel) May 2007 together with synthetic spectra of $11.5 \pm 0.5 \mathrm{kK}$, demonstrating the temperature sensitivity of $K$-band He I lines.

Similarly, a $\beta=3$ law favours a $0.5 \mathrm{kK}$ lower temperature, albeit with a $H$-band bolometric correction consistent to within $0.05 \mathrm{mag}$. These differences lie within the formal uncertainties quoted above, and so do not affect our main conclusions. Alas, no diagnostics are available in the $K$-band from which a determination of the form of the velocity law can be uniquely obtained.

For the 2001 and 2002 epochs, we have retained models from Clark et al. (2003a), but in view of the unresolved He I + Fe II $2.06 \mu \mathrm{m}$ blend and weak He I $2.112 \mu \mathrm{m}$ feature we admit substantially larger uncertainties in temperatures; $12.5 \pm 1.5 \mathrm{kK}$ for Jun. 2001 and $15 \pm 2 \mathrm{kK}$ for Aug. $2002( \pm 0.2 \mathrm{mag}$ in BC). In view of the limitations outlined below, it is apparent that the comparison between physical parameters for AFGL 2298 for 2006 and 2007 epochs is more significant than that between 2001 and 2002 or between UKIRT/CGS4 and VLT/ISAAC datasets.

Specifically for Jun. 2001, the blended emission feature at $2.06 \mu \mathrm{m}$ is significantly stronger than the Fe II $2.04 \mu \mathrm{m}$ line, and indeed comparable in strength to Fe II $2.089 \mu \mathrm{m}$, suggesting that P Cygni He I $2.058 \mu \mathrm{m}$ emission makes a significant contribution to the feature. In contrast He I $2.112 \mu \mathrm{m}$ remains weakly in absorption (albeit blended with Fe II $2.12 \mu \mathrm{m}$ ), from which we estimate a temperature of $12.5 \pm 1.5 \mathrm{kK}$ (recall Fig. 5). Errors are calculated on the basis that $2.06 \mu \mathrm{m}$ is dominated by Fe II at significantly lower temperatures, while negligible $2.112 \mu \mathrm{m}$ He I absorption is predicted at higher temperatures.

For Aug. 2002, a higher stellar temperature of $15.5 \pm 2 \mathrm{kK}$ is suggested on the basis that the $2.06 \mu \mathrm{m}$ blend is now stronger in emission than $2.089 \mu \mathrm{m}$ Fe II, such that it is presumably 
dominated by He I $2.058 \mu \mathrm{m}$. In addition, He I $2.112 \mu \mathrm{m}$ is now observed to be weakly in emission. Similar issues apply regarding errors for the 2002 dataset, although a slightly higher temperature (by $\sim 0.5 \mathrm{kK}$ ) is potentially favoured by the weakness of the Mg II 2.13-2.14 $\mu$ m doublet.

\subsection{Results}

A summary of spectroscopic results is presented in Table 2. We find that the large changes in photometric magnitude between 2001-2007 (e.g. $\Delta J=1.0 \mathrm{mag}$ ) were driven by significant variation in stellar radius (from $160-385 R_{\odot}$ between $2002-2006$ ), albeit with only moderate changes in temperature $-\Delta T_{*} \leq 4.5 \mathrm{kK}$; significantly smaller than observed in the $\mathrm{S}$ Dor excursions of AG Car (Groh et al. 2009). During this period, the mean photospheric expansion velocity was $v_{\text {exp }}=1.25 \mathrm{~km} \mathrm{~s}^{-1}$.

In particular, the photometric fading between 2006-2007 appears to result almost entirely from a reduction in stellar radius from 385 to $310 R_{\odot}\left(v_{\exp }=-1.6 \mathrm{~km} \mathrm{~s}^{-1}\right)$ at near-constant temperature $\left(\Delta T_{*} \sim 0.5 \mathrm{kK}\right)$. This combination of parameters results in a decrease in $L_{\mathrm{bol}}$ for $A F G L 2298$ from 2 to $1.5 \times 10^{6} L_{\odot}$ in less than one year. Such a variation differs from that inferred for both S Dor (Lamers 1995) and AG Car (Groh et al. 2009) in the sense that AFGL 2298 is most luminous while at maximum radius, reversing the behaviour of the other LBVs.

Both terminal wind velocity and (unclumped) mass loss rates determined for AFGL 2298 (Table 2) are comparable to those found for other highly luminous (candidate) LBVs such as the Pistol Star and FMM 362 (Najarro et al. 2009). The maximum mass-loss rate observed for AFGL 2298 is significantly lower than that found for $\eta$ Car and, somewhat surprisingly, the cool B supergiant HDE 316285, which is of a similar temperature but is a factor of $\sim 5$ less luminous (Hillier et al. 2001, 1998). Both these stars support winds with very high performance numbers $\left(\eta\left(=c \dot{M} v_{\infty} / L\right)\right.$ of 18 and 4.5 , respectively; Hillier et al. 1998,2001 ); the lower value for AFGL 2298 ( $\eta \sim$ unity) demonstrating that it's current wind could likely be driven through radiation pressure. The results of our modelling - in particular the comparatively modest mass loss rates - indicate that the changes in physical properties of AFGL 2298 reflect real changes in the stellar temperature and radius.

Finally, our spectroscopic modelling enables us to determine BC's for AFGL 2298 as a function of stellar temperature and hence interpret the photometric lightcurve prior to 2001. In doing so, we find that assuming a $\mathrm{BC} \sim 0 \mathrm{mag}$ (corresponding to $T_{*} \sim 8 \mathrm{kK}$; appropriate for an LBV in the cool state) during the 1996-1999 maximum results in $\log \left(L_{*} / L_{\odot}\right) \sim 6.4$, rising to $\log \left(L_{*} / L_{\odot}\right) \sim 6.6$ for an assumed $T_{*} \sim 11 \mathrm{kK}$, with the former estimate representing a robust lower limit to luminosity at this time. In comparison to the 2007 results, this demonstrates at a $>5 \sigma$ level of significance that the $L_{\mathrm{bol}}$ of AFGL 2298 was not conserved between 1996-2007. We present an updated HR diagram in Fig. 6. At its highest luminosity, AFGL 2298 was one of the brightest stars in the Galaxy, being rivalled and surpassed only by WR 102ka (Barniske et al. 2008) and $\eta$ Car (Hillier et al. 1998).

\section{AFGL 2298 in the context of LBV eruptions}

Our results strongly support the hypothesis that the bolometric luminosity of AFGL 2298 has varied by at least a factor of

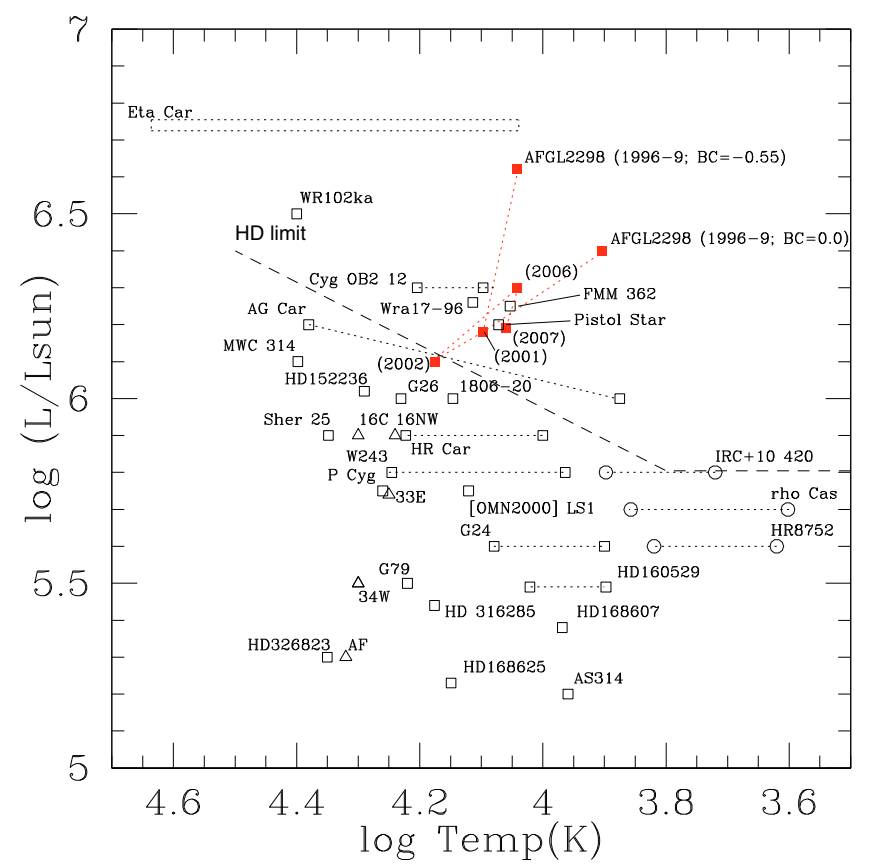

Fig. 6. H-R diagram showing the positions of AFGL 2298 between 1996-2007 in comparison to both confirmed and candidate Galactic LBVs (squares), narrow emission line sources from the Galactic Centre cluster (triangles) and Galactic YHGs (circles). We present two possible locations for AFGL 2298 between 1996-1998 based on temperatures of $8 \mathrm{kK}$ and $11.5 \mathrm{kK}$ (see Sect. 4 for justification). References for luminosity and temperature for individual systems are given in Clark et al. (2005b), with the exception of the Galactic Centre narrow emission line sources (Martins et al. 2007), the Pistol Star and FMM 362 (Najarro et al. 2009), SGR 1806-20 (Bibby et al. 2008), HD 326823 (Marcolino et al. 2007), AG Car (Groh et al. 2009), HR Car (Szeifert et al., in prep.) W243 (Ritchie et al., submitted), WR 102ka (Barniske et al. 2008), [OMN2000] LS1 (Clark et al. 2009) and G24.73+0.69 (Clark et al., submitted).

$\sim 2$ over the past 2 decades (1996-1999 compared to 2002) ${ }^{2}$. Consequently, these variations differ from both the canonical excursions of LBVs to lower temperatures at near constant luminosity, and also the reduction in luminosities inferred for both AG Car and S Dor during such transitions to cooler states, for which the deficit in radiative energy is thought to be due to the requirement to support the expansion of the outer layers of the star.

Given that increases in $L_{\text {bol }}$ for other LBVs have previously only been associated with $\eta$ Car-like giant eruptions, these observations are of considerable interest; more so given the twin suggestions that such behaviour is accompanied by significant mass loss - permitting the transition from Main Sequence to hydrogen depleted Wolf-Rayet and yielding the characteristic LBV ejection nebulae - and may presage the occurrence of a SN (Sect. 1). As such, does the "outburst" of AFGL 2298 permit it to serve as a template system to calibrate other less well observationally constrained events, or are the known non-luminosity conserving eruptions too heterogeneous to allow this?

\footnotetext{
2 In the following discussion we shall assume that the properties of AFGL 2298 in 2001-2002 are those of it in quiescence, despite this being significantly cooler than e.g. AG Car in such a state $(24 \mathrm{kK}$; Groh et al. 2009); if this is incorrect then the duration of the non-luminosity conserving behaviour and the magnitude of such changes will both be lower limits.
} 
Table 3. Summary of the stellar properties of AFGL 2998 and other LBVs observed to undergo giant eruptions.

\begin{tabular}{|c|c|c|c|c|c|c|c|c|}
\hline Star & Epoch & $\begin{array}{c}R_{*} \\
\left(R_{\odot}\right)\end{array}$ & $\begin{array}{c}T_{\text {eff }} \\
(\mathrm{kK})\end{array}$ & $\log \left(L_{*} / L_{\odot}\right)$ & $\mathrm{H} / \mathrm{He}$ & $\begin{array}{c}v_{\infty} \\
\left(\mathrm{km} \mathrm{s}^{-1}\right)\end{array}$ & $\begin{array}{c}\log \dot{M} / \sqrt{f} \\
M_{\odot} \mathrm{yr}^{-1}\end{array}$ & Reference \\
\hline \multirow[t]{5}{*}{ AFGL 2298} & 1996-9 & - & - & $>6.4$ & - & - & - & $\mathrm{a}$ \\
\hline & 2001 Jun. & 262 & 11.7 & 6.2 & 5.0 & 200 & -4.3 & $\mathrm{a}$ \\
\hline & 2002 Aug. & 158 & 10.9 & 6.1 & 5.0 & 200 & -3.9 & $\mathrm{a}$ \\
\hline & 2006 Jun. & 385 & 10.3 & 6.3 & 5.0 & 125 & -4.3 & a \\
\hline & 2007 May & 312 & 10.9 & 6.2 & 5.0 & 125 & -4.4 & $\mathrm{a}$ \\
\hline \multirow[t]{7}{*}{ NGC 2363-V1 } & $<1993$ & - & - & $\leq 5.9$ & - & - & - & $\mathrm{b}$ \\
\hline & 1997 Nov. & 356 & 13.5 & 6.58 & 4.0 & 325 & -3.3 & $\mathrm{~b}, \mathrm{c}$ \\
\hline & 1999 Jul. & 201 & 18.5 & 6.63 & 4.0 & 290 & -3.2 & $b, c$ \\
\hline & 2000 Oct. & 225 & 17.5 & 6.63 & 4.0 & - & -3.3 & $\mathrm{c}, \mathrm{d}$ \\
\hline & 2001 Dec. & 223 & 18.5 & 6.72 & 4.0 & - & -3.5 & $c, d$ \\
\hline & 2003 May & 194 & 20.5 & 6.78 & 4.0 & - & -3.8 & $c, d$ \\
\hline & 2004 May & - & 26.0 & 6.80 & - & - & $<-3.4$ & $\mathrm{c}, \mathrm{d}$ \\
\hline \multirow{4}{*}{ HD 5980} & $1978-80$ & - & 60.5 & 6.4 & - & - & - & $\mathrm{e}$ \\
\hline & 1993 Nov. & - & 52.0 & 6.5 & - & - & - & $\mathrm{e}$ \\
\hline & 1994 Sep. & 280 & 23 & 7.05 & 0.7 & 500 & -3.1 & $\mathrm{~b}$ \\
\hline & 1994 Dec. & 130 & 35 & 6.50 & 2.5 & 600 & -3.0 & $\mathrm{e}$ \\
\hline \multirow[t]{3}{*}{$\eta$ Car } & 1840's & - & - & 7.5 & - & $650-3000$ & $\gtrsim-0.3$ & $\mathrm{f}, \mathrm{g}$ \\
\hline & 1890 's & - & - & - & - & 200 & -1.8 & $\mathrm{~h}, \mathrm{i}$ \\
\hline & 1998.2 & $60-881$ & $35.3-9.2$ & 6.74 & 5.0 & 500 & -3.0 & $\mathrm{j}$ \\
\hline \multirow[t]{2}{*}{ P Cygni } & 1600 's & - & - & - & - & 137 & -1.7 & $\mathrm{k}$ \\
\hline & 1989-92 & 76 & 18.7 & 5.8 & 3.5 & 185 & -4.5 & 1 \\
\hline
\end{tabular}

Note 2. Those parameters that are not directly determined from non-LTE model atmosphere analysis - for example wind parameters derived from present day nebular properties - are given in italics. Due to the extreme mass loss rate for $\eta$ Car a degeneracy is present for the stellar radius and temperature (Hillier et al. 2001). References used in the construction of this table are ${ }^{a}$ Clark et al. (2003a) and this study; ${ }^{b}$ Drissen et al. (2001); ${ }^{c}$ Petit et al. (2005); ${ }^{d}$ Petit et al. (2006); ${ }^{e}$ Koenigsberger et al. (1998) ${ }^{f}$ Humphreys et al. (1999); ${ }^{g}$ Smith et al. (2003); ${ }^{h}$ Smith (2005); ${ }^{i}$ Smith (2008); ${ }^{j}$ Hillier et al. (2001); ${ }^{k}$ Smith \& Hartigan (2006); ${ }^{l}$ Najarro (2001). Volume filling factors, $f$, range from 0.1 (HD 5980 and $\eta$ Car), through 0.3 (NGC 2363-V1) to 0.5 (P Cygni). Time averaged mass loss rates for $\eta$ Car and P Cygni derived from nebular masses are absolute (i.e. $f=1)$. Finally, note that the values of $V_{\infty}$ presented for NGC 2363-V1 in $1997 \& 1999$ are from Drissen et al. $(2001)$, while $R_{*}, T_{\text {eff }}, \log \left(L_{*}\right)$ and $\dot{M}$ are from the updated models presented by Petit et al. (2005).

\subsection{HD 5980 and NGC2363-V1}

Prior to AFGL 2298, two LBVs have been the subject of detailed spectroscopic analysis during the course of a giant eruption: HD 5980 in the SMC and NGC 2363-V1 within NGC 2366, both low metallicity environments. Results of a synthesis of the long-term lightcurves, optical spectroscopy and, for a subset of the data, non-LTE model atmospheric analysis (Drissen et al. 2001; Koenigsberger et al. 1998) are presented in Table 3. A comparison between AFGL 2298, HD 5980 and NGC 2363-V1 reveals that the properties of the three outbursts differ significantly from one another in terms of the duration of the event - 6 months for HD 5980 (e.g. Koenigsberger et al. 1998) to $>7$ yrs for NGC 2363-V1 (Petit et al. 2006) and apparently AFGL 2298. Unless fortuitously located when AFGL 2298 was inaccessible, the sampling frequency of the lightcurve would appear to exclude such a rapid outburst as that of HD 5980, although one could have been missed in NGC 2363-V1 (Petit et al. 2006).

Additionally, significant variance is found between peak luminosity and the evolution of stellar temperature during the outbursts (Table 3). In particular, while the temperature of AFGL 2298 varied by only $\sim 4.5 \mathrm{kK}$ between $2001-2007$, the initial, pre-1997, phase of the outburst of NGC 2363-V1 must have been accompanied by a significant reduction in temperature (for any reasonable progenitor; Drissen et al. 2001), a trend which has since reversed, with temperature increasing by $\sim 13 \mathrm{kK}$ over the following seven years, during which time $L_{\mathrm{bol}}$ has continued to increase. Conversely, the increase in $L_{\text {bol }}$ found for HD 5980 appears to have been solely associated with a rapid and dramatic cooling $(\Delta T \sim 30 \mathrm{kK})$, with the subsequent increase in temperature associated with a corresponding reduction in luminosity (noting that the long term secular changes in brightness prior to this event appear to have occurred at $\sim$ constant $\left.L_{\text {bol }}\right)^{3}$.

Finally, despite the lower metallicity of their host galaxies, the mass loss rates for both HD 5980 and NGC 2363-V1 are significantly higher than those observed for AFGL 2298, although for all three stars they appear to be orders of magnitude lower than those associated with $\eta$ Car and P Cygni in outburst (Table 3).

\subsection{The historic eruptions of $\eta$ Car and $P$ Cygni}

Constraints on the outbursts of both stars may be inferred from their historical lightcurves and current properties of their circumstellar ejecta and are summarised in Table 3, supplemented with the results of non-LTE spectroscopic analyses of their current physical properties. P Cygni was observed to undergo two outbursts of $\sim 6 \mathrm{yr}$ and $\sim 3 \mathrm{yr}$ duration which commenced in $1600 \mathrm{AD}$ and $1655 \mathrm{AD}$ respectively. In both events it was inferred to brighten visually by $2-3 \mathrm{mag}$, with the colour information associated with the latter outburst consistent with a (nonunique) interpretation that the $L_{\mathrm{bol}}$ was also higher at this time

\footnotetext{
3 Our estimates of the stellar temperature of HD 5980 prior to the 1994 June-October outburst were made using the photometric and spectroscopic summary present by Moffat et al. (1998) under the assumption that components $\mathrm{B}$ and $\mathrm{C}$ were of constant brightness (utilising values from Foellmi et al. 2008) and adopting temperatures and bolometric corrections appropriate for WN3h and WN6h stars in the Small Magellanic Cloud from Martins et al. (2009).
} 
Table 4. Summary of the progenitor and peak bolometric luminosities of the extragalactic SNe imposters (top panel) and the dust enshrouded optical transients (lower panel).

\begin{tabular}{lccl}
\hline \hline Transient & \multicolumn{2}{c}{$\log \left(L_{*} / L_{\odot}\right)$} & Reference \\
& progenitor & outburst & \\
\hline SN 1961V & 6.4 & 8.7 & $\mathrm{a}, \mathrm{b}$ \\
SN 1954J & 5.7 & 6.5 & $\mathrm{~b}$ \\
SN 1997bs & $<6.2$ & 7.4 & $\mathrm{c}$ \\
SN 2000ch & 6.2 & 6.9 & $\mathrm{~d}$ \\
\hline NGC 300-OT1 & 4.5 & 6.6 & $\mathrm{e}$ \\
& 4.7 & 7.1 & $\mathrm{f}$ \\
SN 2008S & 4.5 & 7.5 & $\mathrm{~g}, \mathrm{~h}$ \\
\hline
\end{tabular}

Luminosities assume a bolometric correction of $\sim 0$ as appropiate for an F supergiant. References are ${ }^{a}$ Goodrich et al. (1989); ${ }^{b}$ Humphreys et al. (1999); ${ }^{c}$ Van Dyk et al. 2000); ${ }^{d}$ Wagner et al. (2004); ${ }^{e}$ Bond et al. (2009); ${ }^{f}$ Berger et al. (2009); ${ }^{g}$ Smith et al. (2009); ${ }^{h}$ Prieto et al. (2008).

(Lamers \& de Groot 1992). Smith \& Hartigan (2006) report the discovery of a dynamically young $0.1 M_{\odot}$ nebula associated with P Cygni which they attribute to the $1600 \mathrm{AD}$ event; if correct, this would imply a mass loss rate of $\sim 0.02 M_{\odot} \mathrm{yr}^{-1},>2$ orders of magnitude larger than those found for AFGL 2298, HD 5980 or NGC 2363-V1.

In a similar manner, Smith (2005) estimated a comparable time averaged mass loss rate for the 1890's outburst of $\eta$ Car (Table 3). The properties of the star during this outburst are difficult to define due to the uncertain circumstellar extinction, but Humphreys et al. (1999) concluded that it is not necessary to postulate an increase in $L_{\text {bol }}$ during this event. However, this was clearly not the case for the famous and well documented $1840 \mathrm{~s}$ eruption (Table 3 and references therein). While the $\sim 20 \mathrm{yr} \mathrm{du}-$ ration of the giant eruption of $\eta$ Car appears comparable to the ongoing eruption of NGC 2363-V1 (Humphreys et al. 1999), the stellar luminosity and mass loss rate were both extreme; the latter exceeding those of AFGL 2298, NGC 2363-V1 and HD 5980 by over 3 orders of magnitude.

While the wind properties inferred for AFGL 2298 are relatively modest, and in principle consistent with line driving, those inferred for the 1840 s and 1890 s outbursts (and the 1600 s outburst of P Cygni) are high enough that an alternative mechanism is required to power the outflows. Moreover, based on the difference in properties between the two Homunculi and further supported by the recent discovery of a high velocity component of the 1840 s ejecta, Smith $(2005,2008)$ suggest that two different physical mechanisms may have initiated and driven the $1840 \mathrm{~s}$ and 1890s outbursts of $\eta$ Car.

\subsection{The extragalactic SNe imposters}

Finally, we consider the so-called SNe imposters or $\eta$ Car analogues. Humphreys et al. (1999) and Van Dyk (2005) review the nine examples identified prior to 2005; of these 4 have been little studied, while a fifth, SN $2008 \mathrm{~kg}$ appears to be an LBV undergoing a normal LBV excursion and so is not discussed further (e.g. Maund et al. 2006). We summarise the limited information on the progenitor and peak bolometric luminosities of the remaining objects in Table 4. For completeness, we also include two recently discovered optical transients for which a connection with SNe imposters has been proposed (e.g. Bond et al. 2009; Smith et al. 2009), although their dust enshrouded progenitors appear to be of lower mass than known LBVs.
Table 5. Summary of the total mass and nebular expansion velocity of LBV (top panel) and YHG (bottom panel) circumstellar ejecta.

\begin{tabular}{lccl}
\hline \hline Star & $\begin{array}{c}v_{\text {neb }} \\
\left(\mathrm{km} \mathrm{s}^{-1}\right)\end{array}$ & $\begin{array}{c}M \\
\left(M_{\odot}\right)\end{array}$ & Reference \\
\hline$\eta$ Car (Homunculus) & 650 & $>12$ & $\mathrm{a}$ \\
$\quad$ (Little Homunculus) & 200 & 0.1 & $\mathrm{~b}$ \\
P Cygni & 136 & 0.1 & $\mathrm{c}$ \\
Pistol Star & $60-95$ & 11 & $\mathrm{~d}, \mathrm{e}$ \\
AFGL 2298 & 70 & 10 & $\mathrm{f}$ \\
AG Car & 70 & 8.9 & $\mathrm{~g}, \mathrm{~h}$ \\
G79.29+0.46 & 44 & 15 & $\mathrm{i}, \mathrm{j}, \mathrm{k}$ \\
Wra 751 & 26 & 1.7 & $\mathrm{~g}, \mathrm{l}$ \\
HD 168625 & 19 & 2.1 & $\mathrm{~m}, \mathrm{n}$ \\
\hline IRC +10 420 & 27 & 0.24 & $\mathrm{o}$ \\
& 35 & 0.46 & $\mathrm{o}$ \\
HD 179821 & 35 & 4.0 & $\mathrm{o}$ \\
\hline
\end{tabular}

${ }^{a}$ Smith et al. (2003); ${ }^{b}$ Smith (2005); ${ }^{c}$ Smith \& Hartigan (2006); ${ }^{d}$ Figer et al. (1999); ${ }^{e}$ Figer et al. (1998); ${ }^{f}$ Ueta et al. (2001); ${ }^{g}$ Voors et al. (2000a); ${ }^{h}$ Smith et al. (1991); ${ }^{i}$ Higgs et al. (1994); ${ }^{j}$ Waters et al. (1996); ${ }^{k}$ Voors et al. (2000b); ${ }^{l}$ Hutsemekers \& van Drom (1991); ${ }^{m}$ Pasquali et al. (2002); ${ }^{n}$ O'Hara et al. (2003); ${ }^{\circ}$ Castro-Carrizo et al. (2007).

As with P Cygni and $\eta$ Car, the lack of spectroscopic observations for the stars complicates determination of their quiescent and outburst properties; the values derived from the literature in Table 4 typically assume an A/F spectral type in outburst (with the natures of the precursors summarised in Van Dyk et al. 2005, and references therein). However, we note the similarity of the outburst spectrum of SN 1997bs to those of NGC 2363-V1 circa 1997 (van Dyk et al. 2000; Petit et al. 2006), potentially implying an upwards revision for the outburst luminosity to $\log \left(L_{*} / L_{\odot}\right) \sim 7.9$.

If the dusty optical transients SN 2008S and NGC 300-OT1 are super-Eddington outbursts (e.g. Smith et al. 2008) rather than electron capture SNe (Thompson et al. 2009), the mass range for such events will be extended downwards into the 10-20 $M_{\odot}$ regime (e.g. Berger et al. 2009). However, even without the inclusion of these objects, the progenitor and outburst luminosities of the SNe imposters emphasise the diversity of non-luminosity conserving eruptions.

Conclusions over the timescale for such events are difficult due to limited sampling of the lightcurves and possible observational biases introduced by surveys optimised to identify SNe. Nevertheless, the durations of the outbursts of SN 1961v, SN 1954j and SN 1997bs (<1 yr; Humphreys et al. 1999, and refs. therein; van Dyk et al. 2000) all appear significantly shorter than those of $\eta$ Car, NGC 2363-V1 and the behaviour of AFGL 2298 at any stage since 1989 . However the timescales of these outbursts, if not their magnitudes, are comparable to that of HD 5980. The variability of SN 2000ch is characterised by its extreme rapidity ( $\sim 2$ mag. on timescales of a $\sim$ week; Wagner et al. 2004), with similar fluctuations also present during the 1840's giant outburst of $\eta$ Car (Frew 2004); comparable behaviour appears to be absent for AFGL 2298, at least since 2001.

\section{Outbursts and nebular formation}

Given the apparently diverse nature of giant eruptions described in Sect. 5, it is of interest to determine whether the physical properties of LBV ejection nebulae are similarly heterogeneous. In Table 5 we summarise the properties of those nebulae surrounding Galactic LBVs for which both current expansion 
velocity and mass have been determined (with an expanded summary presented in Clark et al. 2003b). In compiling this we note that the nebulae associated with P Cygni and $\eta$ Car are relatively youthful $(<400$ yrs $)$ compared to the dynamical ages of the remaining LBVs $\left(>10^{3} \mathrm{yr}\right.$; references given in caption, Table 5); this will be returned to shortly.

One must also be careful to distinguish those stars which might have ejected their nebulae during a RSG phase, an issue highlighted by Voors et al. (2000a), who found that the dust composition of RSG ejecta is comparable to that of LBV nebulae, suggesting similar conditions during their formation. Assuming evolution at constant $L_{\text {bol }}$ once stars enter the supergiant phase, both observational constraints and theoretical predictions suggest that stars with $\log \left(L_{*} / L_{\odot}\right) \leq 5.8$ will pass through a Red Supergiant(RSG)/Yellow Hypergiant (YHG) phase prior to evolving to higher temperatures (Humphreys \& Davidson 1994; Meynet \& Maeder 2000). Thus, from the stars listed in Table 5, G79.29+0.46, Wra 751 and HD 168625 could all have passed through such a phase.

Given such an hypothesis, it is notable that the mass and velocities of the ejecta associated with the YHGs IRC +10420 and HD 179821, which are thought to be post-RSG objects, are comparable to those of Wra 751 and HD 168625 (although the mass of G79.29+0.46 is significantly higher; Table 5). Assuming that the YHG nebulae continue to expand at their current velocities, in $\sim 5000 \mathrm{yr}$ they would be the same size (and age) as the Wra 751 nebula is today. Additionally, IRC +10 420 currently appears to be evolving to higher temperatures and an early Blue Supergiant/WNL phase (Oudmaijer 1998). At such a point the combined nebulae+stellar system would effectively be indistinguishable from Wra 751. Indeed observations of early Blue Supergiant/WNL stars in Wd1 - which have passed through a YHG phase - show them to be significantly variable as well (Clark et al., in prep.), thus replicating all observational properties of an LBV. Therefore, it is at least possible that the ejecta around (a subset of) the low luminosity LBVs was formed in a previous cool hypergiant phase ${ }^{4}$. In this respect the 2001 outburst of $\rho$ Cas which resulted in a brief phase of extreme mass loss with $\dot{M} \sim 10^{-3} M_{\odot} \mathrm{yr}^{-1}-$ directly comparable to the time averaged mass loss rates inferred for IRC +10 420 and HD 179821 (Castro-Carrizo et al. 2007) - may form a workable paradigm.

Of the stars that are too luminous to have evolved through a RSG phase, there is a notable similarity in the properties of the nebulae of AFGL 2298, AG Car and the Pistol Star (Table 5), while the stars themselves are all of comparable luminosity; highly suggestive of a uniform formation history. In comparison to P Cygni and $\eta$ Car (during the production of the Little Homunculus), it is unclear whether the differences in the nebular expansion velocities observed for AFGL 2298, AG Car and the Pistol Star and, for the first two stars, the time averaged mass loss rates (Sect. 3.2, Voors et al. 2000a) are solely due to observing the nebulae at different evolutionary stages. However, it does appear difficult to reconcile the extreme physical properties present during the formation of the Homunculus - in particular the velocity and mass loss rate - with those of AFGL 2298, AG Car and the Pistol Star.

For example, Langer (2008, priv. comm.) suggested that after several thousand years the rapid expansion of the polar lobes of the Homunculus would reduce their surface brightness below our current detection threshold, leaving the slower moving equatorial material as the sole detectable result of the 1840 s outburst, which by that time would resemble the nebulae around

${ }^{4}$ See Smith (2007) for a counterargument for HD 168625.
AFGL 2298 et al. However, Smith et al. (2003) found that only $10-20 \%\left(\leq 2.5 M_{\odot}\right)$ of the total mass of the Homunculus is located in the equatorial region, whereas the masses of the nebulae in question are $\sim 8.9-11 M_{\odot}$. Thus, at the very least it would appear that the latitudinal distribution of nebular mass would have to differ between these objects; applying the "equatorial" to "polar" mass ratio found for the Homunculus to AFGL 2298, AG Car and the Pistol Star would imply total nebular masses in excess of $\sim 50 M_{\odot}$.

\section{Concluding remarks}

The continued spectroscopic and photometric monitoring of AFGL 2298 clearly indicates that it has been highly variable over the last $\sim 20 \mathrm{yr}$. The magnitude of variability ( $>1.6 \mathrm{mag}$ in the near-IR) is typical of that observed for $L_{\text {bol }}$ conserving outbursts in LBVs. However, the results of non-LTE model atmosphere analysis suggest that unlike normal S Dor-like LBV excursions, which occur at constant luminosity, the $L_{\text {bol }}$ of AFGL 2298 varied during this time, being a factor of $\geq 2$ greater during 1996-1999 in comparison to the photometric minimum of 2001-2002 (indeed, during its 1996-1999 peak it was one of the most luminous stars known in the Galaxy). The changes in luminosity appear to be driven by expansion and contraction of the photosphere at $\sim$ constant temperature. As such they differ from those observed for S Dor and AG Car, where an expansion of the star is accompanied by a significant cooling, resulting in an overall reduction in $L_{\mathrm{bol}}$. Throughout these changes both the wind velocity and mass loss rate of AFGL 2298 were moderate, directly comparable with other highly luminous (candidate) LBVs such as the Pistol Star and FMM 362 (Najarro et al. 2009) and entirely consistent with expectations for a line driven wind.

By comparison to the 2001-2002 minimum, the excess energy radiated during the 1996-1999 photometric maximum requires the star to generate an additional $\sim 10^{47} \mathrm{erg}$. We note that with the exception of the Homunculus, this is $\sim$ comparable to the current kinetic energies of LBV ejecta (determined from the nebular masses and expansion velocities given in Table 5). This value does not include the extra requirement of supporting the extended outer layers of the star against gravity during this period. Without a measurement of the mass of material involved it is difficult to infer the energy budget for this, but simply assuming a similar mass to that suggested for S Dor and AG Car (0.2-0.6 $M_{\odot}$; Lamers 1995; Groh et al. 2009) would require the star to find a further $\sim 10^{47}$ erg over this period.

Due to the renewed interest in the role that non $L_{\text {bol }}$ conserving "Giant" LBV eruptions play in the evolution of massive stars and also their possible role in signalling incipient SNe, we compared the properties of AFGL 2298 to those of other LBV eruptions. From the limited data available we found that the properties of such eruptions appear to be highly heterogeneous, to the extent that it was not possible to identify a characteristic template for such events. Timescale varied from $<0.5-\sim 20 \mathrm{yr}$, with peak luminosities and mass loss rates spanning $>2$ and $>3$ orders of magnitude respectively. Placed in this context, we found that the behaviour of AFGL 2298 defined a lower bound to the properties of LBV eruptions in terms of increase in $L_{\text {bol }}$ and mass loss rate.

Likewise, significant variation was found between the current properties of the ejection nebulae associated with LBVs. While this likely partly reflects observational selection effects introduced by observing nebulae at different stages in their life cycle, we suspect that real differences in the physics of the ejection event may also be involved. For example, a subset of the 
lower luminosity $\left(\log \left(L_{*} / L_{\odot}\right)<5.8\right)$ stars could eject their nebulae in a prior cool hypergiant, rather than the current LBV, phase (e.g. Voors et al. 2000a). We note that a comparison of the disparate properties of the Little and Big Homunculi of $\eta$ Car also led Smith (2005) to suggest that the underlying physical cause of both events differed from one another. Indeed, the mass loss rate of $\eta$ Car during the production of the Homunculus is unrivalled by that of any other LBV, with the exception of the mass loss inferred for the progenitors of SN 2006gy (Agnoletto et al. 2009) and SN 2006tf (Smith et al. 2008).

As such, given the diverse nature of the outbursts, nebulae and, where quantifiable, progenitors, one might consider the possibility that more than one physical process may result in non $L_{\text {bol }}$ conserving eruptions (e.g. Smith 2005), depending on the properties of the underlying (binary?) star. Indeed, a number of different mechanisms have already been proposed to explain Giant eruptions, including hydrodynamical instabilities (Smith \& Owocki 2006), the pulsational pair instability (Woosley et al. 2007), tidal interaction in a binary (Koenigsberger 2008) and binary mergers (Morris \& Podsialowski 2006). If this is the case, then the term Luminous Blue Variable would be more applicable as a phenomenological description of the observational properties of a star, rather than as a description of a unique evolutionary phase.

Acknowledgements. J.S.C. acknowledges support from an RCUK fellowship. AZT-24 observations are made within an agreement between Pulkovo, Rome and Teramo observatories.

\section{References}

Agnoletto, I., Bentti, S., \& Cappellaro, E. 2009, ApJ, 691, 1348 Berger, E., Soderberg, A. M., Chevalier, R. A., et al. 2009, ApJ 699, 1850 Bond, H. E., Bedin, L. R., Bonanos, A. Z., et al. 2009, ApJ, 695, L154 Bibby, J. L., Crowther, P. A., Furness, J. P., \& Clark, J. S. 2008, MNRAS, 386, L23

Barniske, A., Oskinova, L. M., \& Hamann, W.-R. 2008, A\&A, 486, 971

Castro-Carrizo, A., Quintana-Lacaci, G., Bujarrabal, V., Neri, R., \& Alcolea, J. 2007, A\&A, 465, 457

Clark, J. S., Larionov, V. M., Crowther, P. A., Egan, M. P., \& Arkharov, A. 2003a, A\&A, 403, 653

Clark, J. S., Egan, M. P., Crowther, P. A., et al. 2003b, A\&A, 412, 185

Clark, J. S., Negueruela, I., Crowther, P. A., \& Goodwin, S. P. 2005a, A\&A, 434, 949

Clark, J. S., Larionov, V. M., \& Arkharov, A. 2005b, A\&A, 435, 239

Clark, J. S., Davies, B., Najarro, F., et al. 2009, A\&A, 504, 429

Drissen, L., Crowther, P. A., Smith, L. J., et al. 2001, ApJ, 546, 484

Figer, D. F., Najarro, F., Morris, M., et al. 1998, ApJ, 506, 384

Figer, D. F., Morris, M., Geballe, T. R., et al. 1999, ApJ, 525, 759

Foellmi, C., Koenigsberger, G., Georgiev, L., et al. 2008, RMxAA, 44, 3

Frew, D. J. 2004, JAD, 10, 6

Gal-Yam, A., Loeonard D. C., Fox, D. B., et al. 2007, ApJ, 656, 372

van Genderen, A. M. 2001, A\&A, 366, 508

Goodrich, R. W., Stringfellow, G. S., Penrod, G. D., \& Filipenko, A. V. 1989, ApJ, 342, 908

Groh, J. H. 2007, Ph.D. Thesis

Groh, J. H., Hillier, D. J., Damineli, A., et al. 2009, ApJ, 698, 1698

Higgs, L. A., Wendker, H. J., \& Landecker, T. L. 1994, A\&A, 291, 295

Hillier, D. J., \& Miller, D. L. 1998, ApJ, 496, 407
Hillier, D. J., \& Miller, D. L. 1999, ApJ, 519, 354

Hillier, D. J., Crowther, P. A., Najarro, F., \& Fullerton, A. W. 1998, A\&A, 340, 483

Hillier, D. J., Davidson, K., Ishibashi, K., \& Gull, T. 2001, ApJ, 553, 837

Humphreys, R., \& Davidson, K. 1994, PASP, 106, 1025

Humphreys, R., Davidson, K., \& Smith, N. 1999, PASP, 111, 1124

Hutsemekers, D., \& van Drom, E. 1991, A\&A, 251, 620

Jimenez-Esteban, F. M., Garcia-Lario, P., Engels, D., \& Manchado, A. 2006, A\&A, 458, 533

Koenigsberger, G. 2004, RMxAA, 40, 107

Koenigsberger, G. 2008, RMxAC, 33, 108

Koenigsberger, G., Pena, M., Schmutz, W., \& Ayala, S. 1998, ApJ, 499, 889

Kotak, R., \& Vink, J. S. 2006, A\&A, 460, L5

Lamers, H. J. G. L. M. 1987, in Instabilities in Luminous Early-Type Stars, ed. H. J. G. L. M. Lamers, \& C. W. H. de Loore, 99

Lamers, H. J. G. L. M. 1995, in Astrophysical Applications of Stellar Pulsations, ed. R. J. Stobie, P. A. Whitelock, ASP Conf. Ser., 83, 176

Lamers, H. J. G. L. M., \& de Groot, M. 1992, A\&A, 257, 153

Lobel, A., Dupress, A. K., \& Stefanik, R. P. 2003, ApJ, 583, 923

Marcolino, W. L. F., de Araujo, F. X., Lorenz-Martis, S., \& Fernandes, M. B. 2007, AJ, 133, 489

Martins, F., Genzel, R., \& Hillier, D. J. 2007, A\&A, 468, 233

Martins, F., Hillier, D. J., Bouret, J. C., et al. 2009, A\&A, 495, 257

Maund, J. R., Smartt, S. J., \& Kudritzki, R.-P. 2006, MNRAS, 369, 390

Meynet, G., \& Maeder, A. 2000, A\&A, 361, 101

Moffat, A. F. J., Marchenko, S. V., Bartzakos, P., et al. 1998, ApJ, 497, 896

Morris, T., \& Podsiadlowski, Ph. 2006, MNRAS, 365, 2

Najarro, F. 2001, ASPC, 233, 133

Najarro, F., Figer, D. F., Hillier, D. J., Geballe, T. R., \& Kudritzki, R. P. 2009, ApJ, 691, 1816

O’Hara, T. B., Meixner, M., Speck, A. K., Ueta, T., \& Bobrowsky, M. 2003, ApJ, 598,1255

Oudmaijer, R. D. 1998, A\&AS, 129, 541

Pasquali, A., \& Comeron, F. 2002, A\&A, 382, 1005

Pasquali, A., Nota, A., Smith, L. J., et al. 2002, AJ, 124, 1625

Pastorello, A., Samrtt, S. J., Mattila, S., et al. 2007, Nature, 447, 829

Petit, V., Drissen, L., \& Crowther, P. A. 2005, ASPC, 332, 157

Petit, V., Drissen, L., \& Crowther, P. A. 2006, AJ, 132, 1756

Prieto, J. L., Kistler, M. D., Thompson, T. A., et al. 2008, ApJ, 681, L9

Smith, L. J. 1991, in Wolf-Rayet stars and interrelations with other massive stars in galaxies, ed. K. van der Hucht, \& B. Hidayat, IAU Symp., 143, 385

Smith, N. 2005, MNRAS, 357, 1330

Smith, N. 2007, AJ, 133, 1034

Smith, N. 2008, Nature, 455, 201

Smith, N., \& Hartigan, P. 2006, ApJ, 638, 1045

Smith, N., \& Owocki, S. P. 2006, ApJ, 645, L45

Smith, N., Gehrz, R. D., \& Hinz, P. M. 2003, AJ, 125, 1458

Smith, N., Li, W., Foley, R. J., et al. 2007, ApJ, 66, 1116

Smith, N., Cornock, R., Li, W., et al. 2008, ApJ, 686, 467

Smith, N., Ganeshalingham, M., Chornock, R., et al. 2009, ApJ, 697, L49

Spoon, H. W. W., de Koter, A., Sterken, C., Lamers, H. J. G. L. M., \& Stahl, O. 1994, A\&AS, 106, 141

Thompson, T. A., Prieto, J. L., Stanek, K. Z., et al. 2009, ApJ, 705, 1364

Trundle, C., Kotak, R., Vink, J. S., \& Meikle, W. P. S. 2008, A\&A, 483, L47

Ueta, T., Meixner, M., Dayal, A., et al. 2001, ApJ, 548, 1020 (U01)

Umana, G., Buemi, C. S., Trigilio, C., \& Leto, P. 2005, A\&A, 437, L1

Van Dyk, S. D. 2005, ASPC, 332, 47

Van Dyk, S. D., Peng, C. Y., \& King, J. Y. 2000, PASP, 112, 1532

Veen, P. M., van der Hucht, K. A., Williams, P. M., et al. 1998, A\&A, 339, L45

Voors, R. H. M., Waters, L. B. F. M., de Koter, A., et al. 2000a, A\&A, 356, 501

Voors, R. H. M., Geballe, T. R., Waters, L. B. F. M., Najarro, F., \& Lamers,

H. J. G. L. M. 2000b, A\&A, 362, 236

Wagner, R. M., Vrba, F. J., Henden, A. A., et al. 2004, PASP, 116, 326

Waters, L. B. F. M., Izumiura, H., Zaal, P. A., et al. 1996, A\&A, 313, 866

Williams, P. M., Kidger, M. R., \& van der Hucht, K. A. 2001, MNRAS, 324, 156

Woosley, S. E., Blinnikov, S., \& Heger, A. 2007, Nature, 450, 390 